\title{
Amiodarone induced ischemic optic neuropathy: A case
}

\section{report}

\author{
Nor Ismail, Martin Royle, Mark Payne, Amjad Syed and Abdalazeem Ibrahem* \\ BCUHB, Ysbyty Gwynedd, Bangor, LL57 2PW, UK
}

\section{Introduction}

Amiodarone is an anti-arhythmic drug that is used commonly to treat ventricular and supra ventricular arrhythmia [1]. Its use has increased over the past years due to its efficacy at restoring sinus rhythm and its use in cardiac resuscitation [2]. Despite its increasing popularity, amiodarone has been associated with various side effects. The side effects of amiodarone are due to its cationic amphophilic properties which allow interactions with lipid compounds which accumulate in multiple tissues [3]. Among the tissues affected is the optic nerve which can give rise to optic neuropathy. The reduction in visual acuity has been reported to be significant and permanent, which massively effects patient's quality of life. Nevertheless, making the diagnosis of amiodarone induced optic neuropathy (AON) is challenging due to the lack of diagnostic tests and is mostly a clinical diagnosis. Furthermore, the alternative differential diagnosis of non-arteritic ischaemic optic neuropathy (NAION) is similarly made on clinical grounds. This makes the diagnosis of AON highly dependent on the typical clinical features seen in AON which makes it a diagnostic challenge when one or more atypical features are present in the clinical presentation. In this case, we present the diagnostic challenge in a case of amiodarone induced ischemic optic neuropathy (AON) which occurred in a 55-year-old man over an atypically shorter time course.

\section{Case Report}

A 55-year-old man with a history of obesity and high alcohol intake presented to his general practitioner (GP) with palpitations. An electrocardiogram (ECG) confirmed atrial fibrillation (AF). He was started on flecainide $50 \mathrm{mg}$ twice a day and rivaroxaban $20 \mathrm{mg}$ daily and was successfully electrically cardioverted. Despite continuing on flecainide, 2 months later, he reverted back to AF. Given he was symptomatic, flecainide was stopped, and the patient was commenced on amiodarone $200 \mathrm{mg}$ three times a day (a re-ducing regimen), and a repeat electrical cardioversion was arranged.

On review in Cardiology Clinic, the patient complained of gradual onset blurring of vision in both eyes which started 9 weeks after commencing on Amiodarone. His cranial nerves and neurology examination were normal, and he was referred to the ophthalmologists. Amiodarone was discontinued after a close discussion between cardiologist and ophthalmologist.

The patient was reviewed by the ophthalmologist 2 weeks after discontinuing Amiodarone where he re-ported that his vision had improved slightly since discontinuing the drug. His visual acquity was $6 / 24$ in the right eye and 6/18 in the left eye. An optic computed tomography (Figures la and 2a) shows loss of nerve fiber and that the cup disc ratio was unable to be commented on due to this. The optic discs were swollen bilaterally on fundoscopy (Figures $1 b$ and $2 b$ ). The visual fields (Figures $3 \mathrm{a}$ and $3 \mathrm{~b}$ ) showed that the patient had peripheral and generalized defect without altitudinal changes.

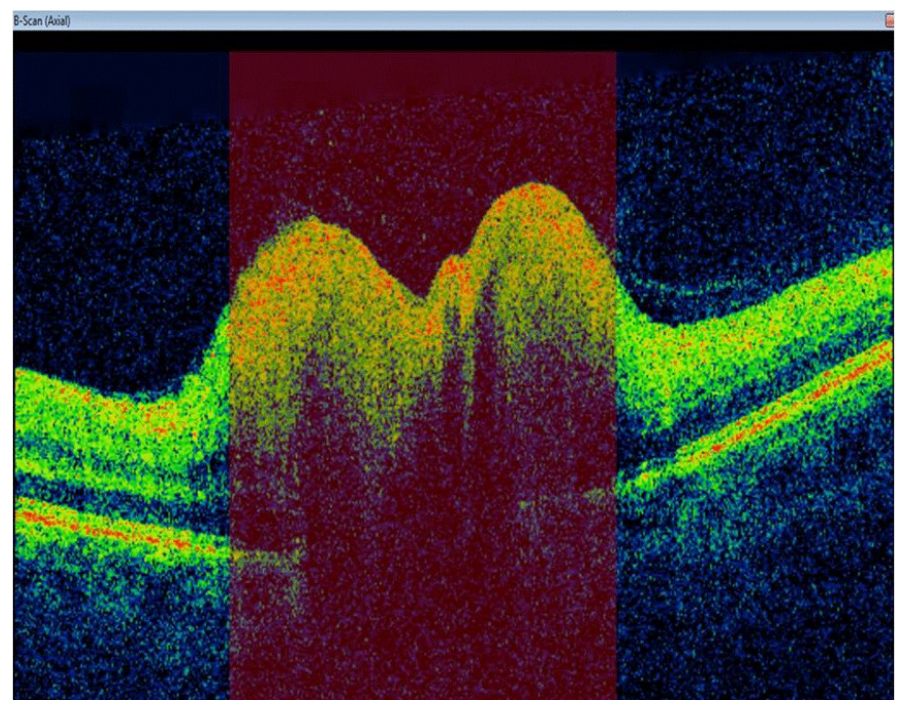

Figure 1a. Optic Computed Tomography of the right eye showing thinning of nerve fiber

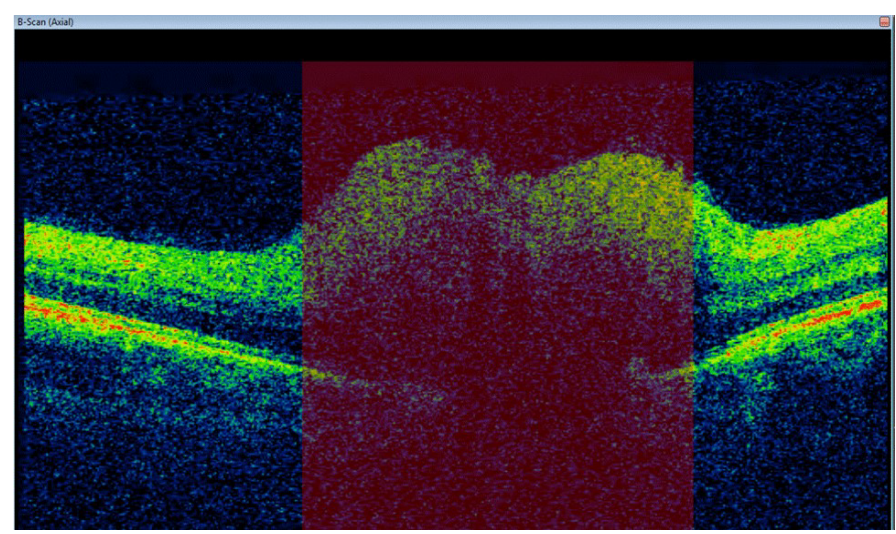

Figure 1b. Optic Computed Tomography of the left eye showing thinning of nerve fiber

${ }^{\star}$ Correspondence to: Abdalazeem Ibrahem, BCUHB, Ysbyty Gwynedd, Bangor, LL57 2PW, UK, E-mail: abdalazeemibrahem@yahoo.co.uk

Received: February 09, 2021; Accepted: February 17, 2021; Published: February 26,2021 


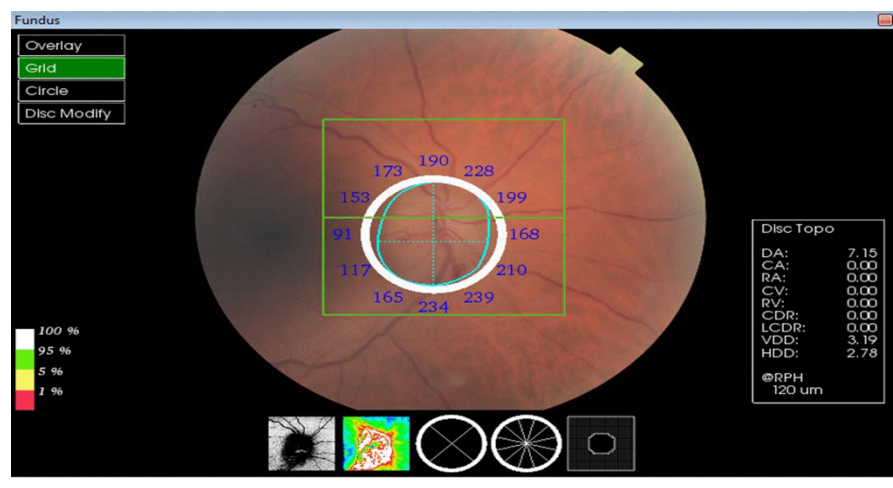

Figure 2a. Fundoscopy of the right eye showing optic disc swelling

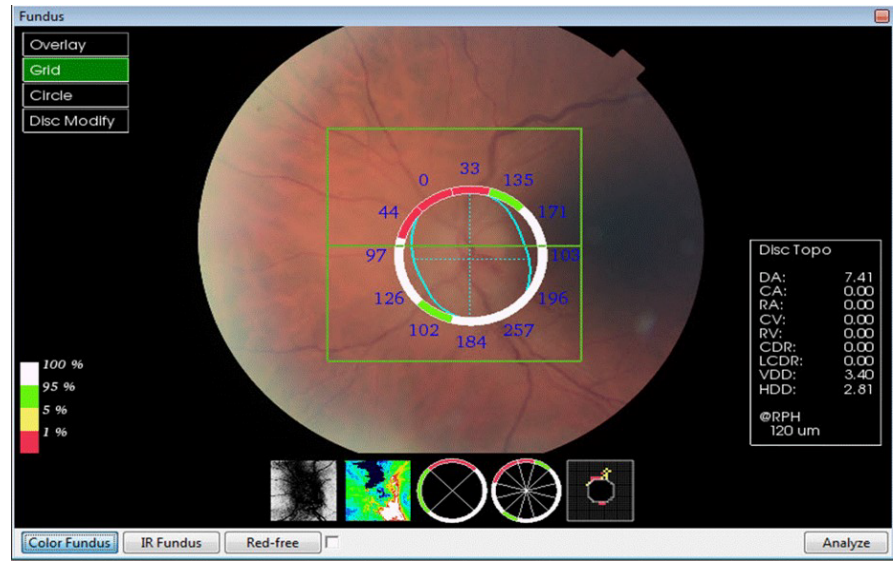

Figure 2b. Fundoscopy of the left eye showing disc swelling
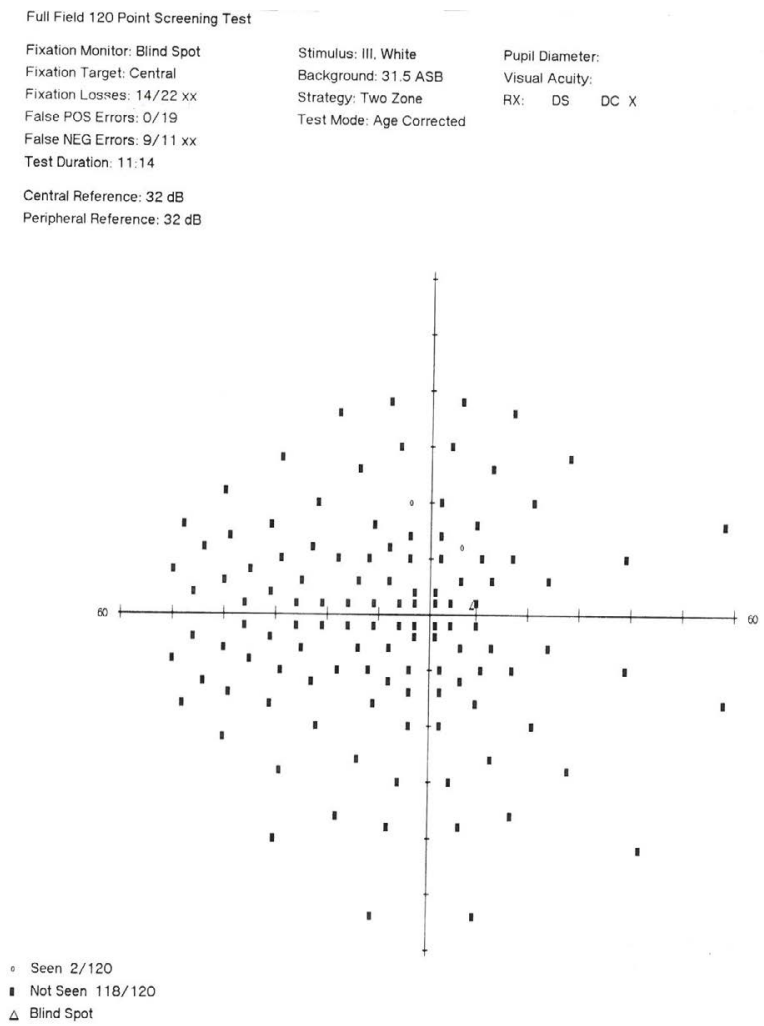

Figure 3a. Visual field right eye showing peripheral and generalized defect

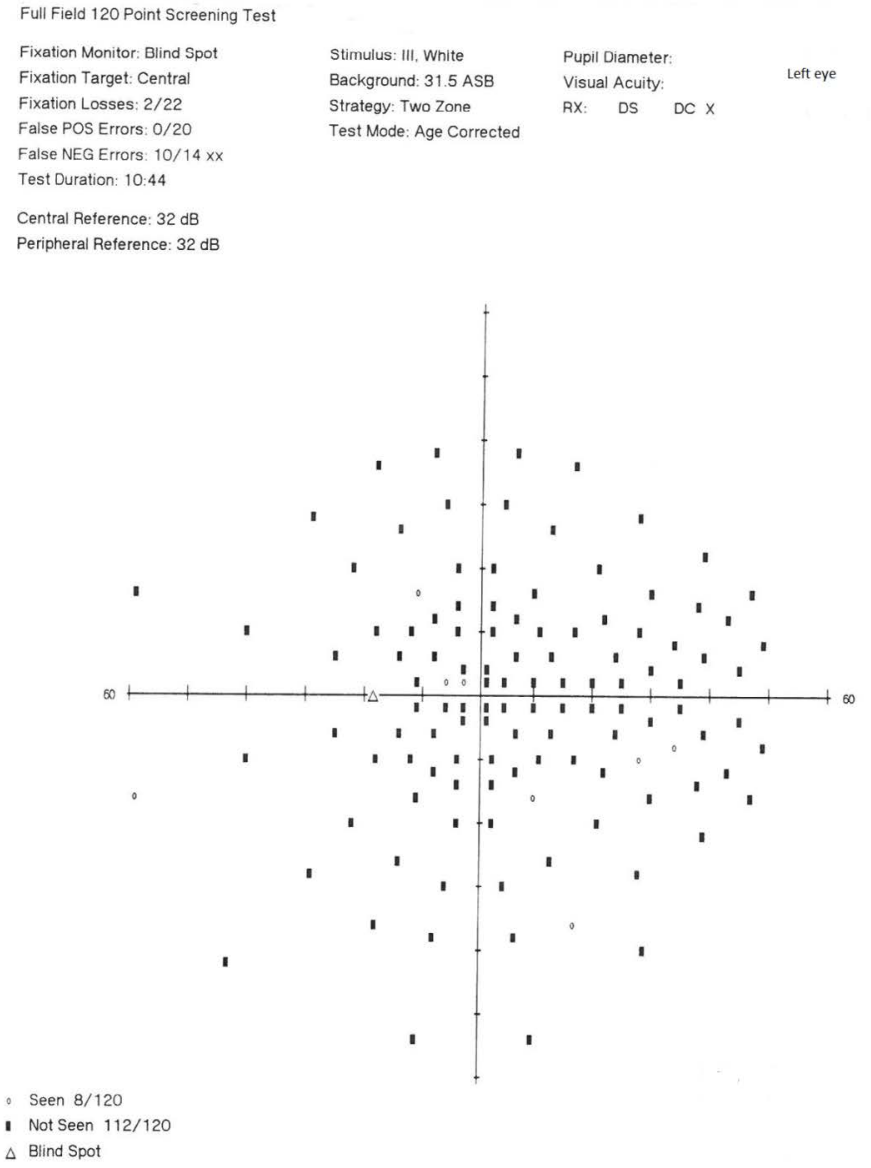

Figure 3b. Visual field left eye showing peripheral and generalized defect

The ophthalmologist referred the patient to the respiratory team to investigate for sleep apnea where he was found to have severe sleep apnea and was commenced on overnight CPAP.

A CT head, MRI head, lumbar puncture and blood for FBC, U+E, CRP were normal.

Given the evidence of swollen disc on fundoscopy, symptoms occurring after the commencement of amiodarone and slow resolution after its discontinuation, and peripheral defects on the visual fields, the most plausible explanation was that the patient had sustained AON.

The patient was referred to our local tertiary cardiac centre for catheter ablation of his AF. Regular follow up in Ophthalmology Clinic was arranged.

Visual acuity tests performed in the subsequent weeks showed worsening of the patient's visual acuity in the first 3 weeks after the discontinuation of Amiodarone and improved then on. The fundoscopy on sub-sequent weeks showed persistent bilateral discs swelling with no resolution up to 16 weeks of review.

\section{Discussion}

The diagnosis of amiodarone-induced optic neuropathy (AON) is difficult to make and is a clinical diagnosis. There is a lack of causal evidence between the use of amiodarone and the occurrence of optic neuropathy in the literature [4], difficulty in distinguishing AON from NAION, and, in this patient an early time of onset of symptoms after the commencement of amiodarone. 
The causal relationship between the use of amiodarone and optic neuropathy has been largely postulat-ed rather than based on empirical evidence [4]. The theory behind the toxicity reaction associated with amiodarone is related to its cationic ampophilic properties which allow the formation of intracellular drug-lipid complexes that are nondigestible and consequently accumulate in lysosomes as intracytoplasmic inclusion bodies [5]. Microscopic examination of optic nerves in patients with $\mathrm{AON}$ has shown multiple lamellated inclusion bodies in large axons of the optic nerve [3] which either mechanically or biochemically decreases axoplasmic flow causing swelling of the optic head and consequently optic neu-ropathy [6]. Despite these subclinical structural changes in the optic nerve, it is unclear whether this can cause the optic head swelling or the visual changes in optic neuropathy [7].

Distinguishing AON from NAION has always been a subject of controversy. There have been many at-tempts in the literature to distinguish these two diagnoses. Typically, AON is characterized by an insidi-ous onset optic disc swelling occurring within 12 months of initiating amiodarone and resolution of optic disc swelling occurring one to eight months after discontinuation of drug [8]. NAION on the other hand typically presents acutely with unilateral visual loss and resolution of optic disc swelling within two to four weeks of onset of symptoms [8]. Despite the typical presentation of AON in this patient, i.e. insidi-ous onset of bilateral disc swelling and optic disc swelling still present 3 months after discontinuation of the drug, there has been reported variation in the clinical presentation of AON. A study on the clinical spectrum of AON found that around $30 \%$ of reported AON occur acutely with unilateral visual loss [9]. Similarly, there are also variations in the presentation of NAION in which there have been reported cases where optic neuropathy occurred bilaterally. However, bilateral cases of NAION usually occur sequentially with a 25thpercentile time to bilaterally of 32.4 months [10]. Simultaneous bilateral NAION is extremely rare [11]. The other difficulty in distinguishing these two diagnoses is the fact that most pa-tients on amiodarone have the risk factors for NAION [9] and that the optic neuropathy may simply be NAION which occurred whilst patients were coincidentally on amiodarone. The risk factors for NAION include a previous attack of NAION in one eye, sleep apnea, small optic cup-disc ratio, age more than 50 years and hypertension [9]. This patient's risk factors for NAION include age more than 50 years and a history of sleep apnea. Regardless of the risk factors for NAION in this patient, the fact that this patient had a bilateral simultaneous onset of symptoms and presented after the commencement of amiodarone and with slow resolution of disc swelling after discontinuation of amiodarone makes the diagnoses of AON more likely.

Finally, the onset of symptoms following commencement of amiodarone in this patient is earlier than in most reported cases. The literature largely describes the onset of visual loss to be within 12 months of starting amiodarone with a median time of 6 months [9]. This patient presented after only 9 weeks of starting amiodarone. A review of all published cases of AON found that optic neuropathy can occur as early as 72 hours after the commencement of IV amiodarone [9]. This patients time of onset of blurring of vision is in keeping with a diagnosis of AON. Furthermore, the progression of visual acuity during follow-up showed that the patient's visual acuity actually worsened in the first 3 weeks following discontinuation of amiodarone and then slowly improved. This progression in visual acuity is still in keeping with a diagnosis of AON as the drug has a long half-life and patients can develop symptoms of toxicity as long as 4 weeks after discontinuation of drug [9].

In summary, the diagnosis of amiodarone-induced optic neuropathy (AION) is a difficult diagnosis to make and should be considered carefully because it has important implications for patient care and for informed consent. As well as being a potential cause for medicolegal suits, this diagnosis also prefers the discontinuation of amiodarone, even when other antiarrhythmic options are limited. This will pose a clinical dilemma which would require a close discussion between cardiologist and ophthalmologist to decide on best patient management.

\section{References}

1. Nagra PK, Foroozan R, Savino PJ, Castillo I, Sergott RC (2003) Amiodarone-induced optic neuropathy. Br J Ophthalmol 87: 420-422.

2. Wolkove N, Baltzan M (2009) Amiodarone Pulmonary Toxicity. Can Respir J 16: 4348. [Crossref]

3. Mansoor AM, Puklin JE, O'Grady (1988) Optic nerve ultrastructural following amiodarone therapy. J Clin Neurooptahlmol 8: 231-237. [Crossref]

4. Murphy MA, Murphy JF (2005) Amiodarone and Optic Neuropathy: The Heart of the Matter. J Neuro-Ophthalmol 25: 232-236. [Crossref]

5. Burkhardt H, Drenched D, Ullmann-Rauch R (1978) Amiodarone induced the lipidosis-like alteration in ocular tissues of the rat. Grief as Arch Klin Exp Ophthalmol 207: 91-96. [Crossref]

6. Garrett SN, Keaney JJ, Schiffman JS (1988) Amiodarone Optic Neuropathy. J Clin Neuro-Ophthalmol 8: 105-110.

7. Passman RS, Bennet CL, Purpura JM, Kapur R, Johnson LN, et al. (2012) Amiodaroneassociated Optic Neuropathy: A Critical Review. Am J Med 125: 447-453. [Crossref]

8. Macaluso DC, Shults WT, Frauenfelder FT (1999) Features of amiodarone-induced optic neuropathy. Am J Ophthalmol 127: 610-612. [Crossref]

9. Johnson LN, Krohel GB, Thomas ER (2004) The clinical Spectrum of Amiodaroneassociated Optic Neu-ropathy. J Natl Med Assoc 96: 1477-1491. [Crossref]

10. Beri M, Klugman MR, Kohler JA, Hayreh SS (1987) Anterior ischaemic optic neuropathy: VII. Incidence of bilaterality and various influencing factors. Opthalmology 94: 1020-1028. [Crossref]

11. Tarantini A, Faraoni A, Menchini F, Lanzetta P (2012) Bilateral Simultaneous Nonarteritic Anterior Is-chaemic Optic Neuropathy after ingestion of Sildenafil for Erectile dysfunction. Case Rep Med 2012: 747658. [Crossref]

Copyright: (C2021 Ismail N. This is an open-access article distributed under the terms of the Creative Commons Attribution License, which permits unrestricted use, distribution, and reproduction in any medium, provided the original author and source are credited. 